\title{
Erythropoietin protects the inner blood-retinal barrier by inhibiting microglia phagocytosis via Src/Akt/cofilin signalling in experimental diabetic retinopathy
}

\author{
Hai Xie ${ }^{1} \cdot$ Chaoyang Zhang ${ }^{1} \cdot$ Dandan Liu $^{1} \cdot$ Qian Yang $^{1} \cdot$ Lei Tang $^{1} \cdot$ Tianqin Wang $^{2} \cdot$ Haibin Tian $^{1} \cdot$ Lixia Lu $^{1}$.

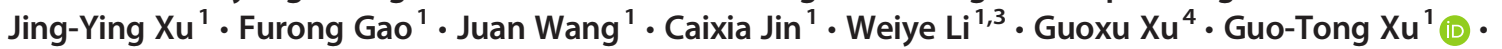 \\ Jingfa Zhang ${ }^{1,5,6,7,8,9}$
}

Received: 24 April 2020 / Accepted: 19 August 2020 / Published online: 26 October 2020

(C) Springer-Verlag GmbH Germany, part of Springer Nature 2020

\begin{abstract}
Aims/hypothesis Microglial activation in diabetic retinopathy and the protective effect of erythropoietin (EPO) have been extensively studied. However, the regulation of microglia in the retina and its relationship to inner blood-retinal barrier (iBRB) maintenance have not been fully characterised. In this study, we investigated the role of microglia in iBRB breakdown in diabetic retinopathy and the protective effects of EPO in this context.

Methods Male Sprague Dawley rats were injected intraperitoneally with streptozotocin (STZ) to establish the experimental model of diabetes. At $2 \mathrm{~h}$ after STZ injection, the right and left eyes were injected intravitreally with EPO (16 mU/eye, $2 \mu \mathrm{l}$ ) and an equivalent volume of normal saline $(\mathrm{NaCl} 154 \mathrm{mmol} / \mathrm{l})$, respectively. The rats were killed at 2 or 8 weeks after diabetes onset. Microglia activation was detected by ionised calcium binding adaptor molecule (IBA)-1 immunolabelling. Leakage of the iBRB was evaluated by albumin staining and FITC-dextran permeability assay. BV $_{2}$ cells and primary rat microglia under hypoxic conditions were used to model microglial activation in diabetic retinopathy. Phagocytosis was examined by confocal microscopy in flat-mounted retina preparations and in microglia and endothelial cell cocultures. Protein levels of IBA-1, CD11b, complement component $1 \mathrm{r}(\mathrm{C} 1 \mathrm{r})$, and Src/Akt/cofilin signalling pathway components were assessed by western blotting.

Results In diabetic rat retinas, phagocytosis of endothelial cells by activated microglia was observed at 8 weeks, resulting in an increased number of acellular capillaries (increased by $426.5 \%$ ) and albumin leakage. Under hypoxic conditions, activated microglia transmigrated to the opposite membrane of the transwell, where they disrupted the endothelial cell monolayer by engulfing endothelial cells. The activation and phagocytic activity of microglia was blocked by intravitreal injection of EPO. In vitro, IBA-1, CD11b and C1r protein levels were increased by $50.9 \%, 170.0 \%$ and $135.5 \%$, respectively, by hypoxia, whereas
\end{abstract}

Jingfa Zhang and Guo-Tong Xu contributed equally to this study.

Electronic supplementary material The online version of this article (https://doi.org/10.1007/s00125-020-05299-x) contains peer-reviewed but unedited supplementary material, which is available to authorised users.

Jingfa Zhang

jingfazhang@tongji.edu.cn

Guo-Tong Xu

gtxu@ tongji.edu.cn

1 Tongji Eye Institute, Tongji University School of Medicine, Shanghai, China

2 Department of Ophthalmology, Renji Hospital, Shanghai Jiao Tong University, Shanghai, China

3 Department of Ophthalmology, Drexel University College of Medicine, Philadelphia, PA, USA

4 Department of Ophthalmology, the Second Affiliated Hospital of Soochow University, Suzhou, China
5 Department of Ophthalmology, Shanghai General Hospital (Shanghai First People's Hospital), Shanghai Jiao Tong University, Shanghai, China

6 National Clinical Research Center for Eye Diseases, Shanghai, China

7 Shanghai Key Laboratory of Ocular Fundus Diseases, Shanghai, China

8 Shanghai Engineering Center for Visual Science and Photomedicine, Shanghai, China

9 Shanghai Engineering Center for Precise Diagnosis and Treatment of Eye Diseases, Shanghai, China 


\section{Research in context}

\section{What is already known about this subject?}

- Microglia are activated in diabetic retinopathy

- Inner blood-retinal barrier (iBRB) breakdown happens early in diabetic retinopathy

- Erythropoietin (EPO) protects the retina from insults of diabetes

What is the key question?

- How do activated microglia damage endothelial cells in diabetic retinopathy and can EPO prevent this through supressing the activation of microglia to protect the iBRB?

What are the new findings?

- Activated microglia can penetrate the basement membrane of retinal blood vessels and phagocytose the endothelial cells, resulting in iBRB breakdown

- $\quad$ EPO prevented iBRB disruption through inhibiting microglial activation and phagocytosis by activating the Src/Akt/cofilin signalling pathway in microglia

How might this impact on clinical practice in the foreseeable future?

- Our findings indicate that EPO, as a multifunctional molecule, is a potential candidate for the treatment of diabetic macular oedema in early diabetic retinopathy and the regulation of microglia might represent an alternative approach for the treatment of diabetic retinopathy

the phosphorylated proteins of Src/Akt/cofilin signalling pathway components were decreased by $74.2 \%, 47.8 \%$ and $39.7 \%$, respectively, compared with the control; EPO treatment abrogated these changes.

Conclusions/interpretation In experimental diabetic retinopathy, activated microglia penetrate the basement membrane of the $\mathrm{iBRB}$ and engulf endothelial cells, leading to iBRB breakdown. EPO exerts a protective effect that preserves iBRB integrity via activation of Src/Akt/cofilin signalling in microglia, as demonstrated in vitro. These data support a causal role for activated microglia in iBRB breakdown and highlight the therapeutic potential of EPO for the treatment of diabetic retinopathy.

Keywords Diabetic retinopathy $\cdot$ Erythropoietin $\cdot$ Inner blood-retinal barrier $\cdot$ Microglia $\cdot$ Retinal vascular endothelial cell

$\begin{array}{ll}\text { Abbreviations } \\ \text { AC } & \text { Acellular capillary } \\ \text { C1r } & \text { Complement component 1r } \\ \text { EPO } & \text { Erythropoietin } \\ \text { HRMEC } & \text { Human retinal microvascular endothelial cell } \\ \text { IBA-1 } & \text { Ionised calcium binding adaptor molecule-1 } \\ \text { iBRB } & \text { Inner blood-retinal barrier } \\ \text { IB4 } & \text { Isolectin B4 } \\ \text { PI } & \text { Propidium iodide } \\ \text { RM } & \text { Rat primary microglia } \\ \text { STZ } & \text { Streptozotocin } \\ \text { VEGF } & \text { Vascular endothelial growth factor }\end{array}$

\section{Introduction}

Diabetic retinopathy, a leading cause of blindness in the working-age population [1-3], is considered as both microangiopathy and neuropathy that affects all retinal cells.
Neurovascular unit injury is also implicated in the aetiology of diabetic retinopathy $[4,5]$. Glial activation is thought to be the first event in the pathogenesis of diabetic retinopathy [6, 7].

Microglia are resident cells of the retina that can differentiate from monocytes $[8,9]$. Under normal conditions, microglia have a ramified morphology and screen the retina by virtue of their long processes [10]; when the microenvironment is perturbed, they become activated and assume an amoeboid form $[11,12]$. Activated microglia are involved in inflammation, migrating from the inner to outer retina and releasing proinflammatory cytokines such as TNF- $\alpha$ and IL-1 $\beta[13$, 14]. The observed perivascular clustering of microglia and fibrinogen deposition in diabetic retinopathy suggests a relationship between microglial activation and disruption of the inner blood-retinal barrier (iBRB) [15].

Erythropoietin (EPO), besides stimulating erythrocyte production, has multiple functions, including neurotrophic activity in ischaemic damage and stroke, Parkinson's disease and retinal degeneration [16]. We previously evaluated the 
efficacy, pharmacokinetics, toxicity and mechanism of action of EPO in experimental diabetic retinopathy [17-19] and found that it has protective effects in retinal neurons [20], microvascular endothelium [19], retinal pigment epithelium (RPE) [21] and Müller cells [22].

In an off-label clinical study, five patients with diffuse diabetic macular oedema who were unresponsive to standard treatments showed improvement upon intravitreal EPO administration. EPO reduced macular oedema and improved visual acuity. Interestingly, in some patients, hard exudates decreased and even disappeared, possibly as a result of phagocytosis by microglia [23].

Microglial activation in diabetic retinopathy has both positive and negative effects [24]. While the clearance of debris is beneficial, activated microglia can also cause damage to normal retina, including the vascular endothelium. We speculated that under diabetic conditions, leaked albumin resulting from iBRB breakdown stimulates microglial migration to the injury site for clearance of hard exudate; however, increased phagocytic activity of the microglia damages normal retinal vascular endothelial cells, thereby exacerbating iBRB breakdown, increasing the number of acellular capillaries (ACs), and leading to further leakage from blood vessels. We also hypothesised that EPO treatment could protect vascular endothelial cells from damage caused by activated microglia.

\section{Methods}

\section{Animal model of diabetic retinopathy and intravitreal EPO} injection All animal procedures were carried out in accordance with the Association for Research in Vision and Ophthalmology (ARVO) Statement for Use of Animals in Ophthalmic and Vision Research and the US National Research Council Guide for the Care and Use of Animals. The protocol was approved by the ethics committee for animal experiments of Tongji University (permit number: TJHBLAC-2020-06). Male Sprague Dawley rats weighing 120-160 g were purchased from Shanghai Laboratory Animal Center (Chinese Academy of Sciences, Shanghai, China) and maintained on a 12:12-h light/dark cycle with free access to food and water. Sprague Dawley rats were randomly assigned to be made diabetic. To establish diabetes, the rats were fasted for $24 \mathrm{~h}$ and i.p. injected with streptozotocin (STZ, $60 \mathrm{mg} / \mathrm{kg}$ body weight in citric acid buffer); control animals were injected with the same volume of citric acid buffer [17]. Information on blood glucose level and body weight for the validation of diabetes establishment after STZ injection is provided in electronic supplementary material (ESM) Table 1. Animals receiving STZ were declared diabetic when their blood glucose exceeded $13.9 \mathrm{mmol} / \mathrm{l}(250 \mathrm{mg} / \mathrm{dl})$ for 3 consecutive days. The rats were excluded from the experiment if they failed to develop diabetes.
At $2 \mathrm{~h}$ after STZ injection, the right eye of diabetic rats was intravitreally injected with EPO (16 mU/eye, $2 \mu \mathrm{l}$; catalogue no. 286-EP; R\&D, MN, USA) (referred to as the D+E group in the figures), while the left eye was injected with the equivalent volume of normal saline $(\mathrm{NaCl} 154 \mathrm{mmol} / \mathrm{l}$; referred to as the D group). Intravitreal injection of EPO was repeated every month, based on our previous work [17]. Age-matched normal control rats were left untreated. Rats were killed at either 2 or 8 weeks after diabetes onset.

$\mathrm{BV}_{2}$ cells, primary human retinal microvascular endothelial cells and rat primary microglia cultures $\mathrm{The}^{\mathrm{B}} \mathrm{BV}_{2}$ mouse microglia cell line, purchased from American Type Culture Collection (Manassas, VA, USA), was cultured in highglucose (25 mmol/l) DMEM containing 10\% (vol./vol.) FBS (catalogue no. 10091148; Gibco, NY, USA) and 1\% (vol./ vol.) penicillin/streptomycin (catalogue no. 15140122; Invitrogen, $\mathrm{CA}, \mathrm{USA}$ ) at $37^{\circ} \mathrm{C}$ and $5 \% \mathrm{CO}_{2}$ in a humidified incubator. After $24 \mathrm{~h}$, the cells were divided into three groups: normal control, hypoxia treatment and hypoxia+EPO treatment. The latter two groups were incubated in a hypoxic workstation (Whitley H35 Hypoxystation; Don Whitley Scientific, Bingley, UK) with $1 \% \mathrm{O}_{2}$ without or with EPO $(1 \mathrm{U} / \mathrm{ml})$ treatment.

To investigate the mechanism of action of $\mathrm{EPO}, \mathrm{BV}_{2}$ cells were pretreated with $0.5 \mu \mathrm{mol} / 1 \mathrm{Src}$ family activator (catalogue no. sc-3052; Santa Cruz Biotechnology, CA, USA), $10 \mu \mathrm{mol} / 1$ Akt activator SC79 (catalogue no. S7863; Selleck, TX, USA) or $10 \mu \mathrm{mol} / 1$ Akt inhibitor MK2206 (catalogue no. S1078; Selleck) for $30 \mathrm{~min}$ to $1 \mathrm{~h}$ before exposure to hypoxia.

Human retinal microvascular endothelial cells (HRMECs; Sciencell Research Laboratories, Carlsbad, CA, USA) were cultured in endothelial culture medium (catalogue no. 1001; Sciencell) containing 5\% (vol./vol.) FBS (catalogue no. 0025; Sciencell), $1 \%$ (vol./vol.) endothelial cell growth supplement (catalogue no. 1052; Sciencell) and 1\% penicillin/ streptomycin solution (catalogue no. 0503; Sciencell) at $37^{\circ} \mathrm{C}$ and $5 \% \mathrm{CO}_{2}$ in a humidified incubator.

Rat primary microglia (RM) (catalogue no. R1900; Sciencell) were cultured in Microglia Medium (catalogue no. 1901; Sciencell) supplemented with 5\% FBS (catalogue no. 0025; Sciencell), 1\% (vol./vol.) Microglia Growth Supplement (catalogue no. 1952; Sciencell), and 1\% penicillin/streptomycin solution (catalogue no. 0503; Sciencell) at $37^{\circ} \mathrm{C}$ and $5 \% \mathrm{CO}_{2}$ in a humidified incubator.

$\mathrm{BV}_{2}$ cells spontaneously detached from the culture dish after incubation under the hypoxic condition, and both adherent and detached cells were separately collected for assays.

To mimic the interaction between microglia and endothelial cells in diabetic retinopathy, we established a $\mathrm{BV}_{2}$ cell/HRMEC coculture system (Fig. 6a). The basal side of the inverted transwell membrane was pre-coated with fibronectin to simulate the basement membrane of capillaries, and the endothelial cells $\left(1 \times 10^{4} /\right.$ well $)$ were seeded and cultured in endothelial cell 
medium until they reached confluence. $\mathrm{BV}_{2}$ cells $\left(1 \times 10^{3} /\right.$ well $)$ were seeded on the apical side of the transwell membrane and cultured in the high-glucose medium. The coculture system was maintained under normoxic or hypoxic conditions for $9 \mathrm{~h}$. Microglial migration and phagocytic activity were examined under a confocal microscope (A1 R HD25; Nikon, Tokyo, Japan). We also established a RM/HRMEC coculture system, for which the time of incubation under normoxic or hypoxic conditions was extended to $48 \mathrm{~h}$ based on the observations for RM activation (see ESM for further details).

Immunofluorescence analysis Eyes were fixed in 2\% (wt/vol.) paraformaldehyde for $1 \mathrm{~h}$ at $4^{\circ} \mathrm{C}$, and anterior segments including the cornea, iris and lens were removed. After dehydration in a graded series of sucrose solutions (wt/vol.; 10 $30 \%$ ), the eye cups were embedded in optimal cutting temperature compound (Sakura Finetek, Tokyo, Japan) and stored at $-80^{\circ} \mathrm{C}$ until sectioning. Frozen serial sections (15- $\mu \mathrm{m}$ thick) were cut on a cryostat microtome (Leica, Wetzlar, Germany).

For immunofluorescence labelling, cryosections were fixed in $2 \%$ paraformaldehyde for $2 \mathrm{~h}$ and permeabilised with blocking/permeabilisation solution composed of $1 \%(\mathrm{wt} /$ vol.) BSA and $0.1 \%$ (vol./vol.) Triton X-100 in $1 \times$ PBS. After blocking in the same solution for $1 \mathrm{~h}$ at room temperature, the sections were incubated overnight at $4^{\circ} \mathrm{C}$ with rabbit anti-IBA-1 antibody (1:500, catalogue no. 019-19741; WAKO, Osaka, Japan). After washing three times with $1 \times$ PBS for 10 min each, the sections were incubated with donkey anti-rabbit IgG H\&L (Alexa Fluor 555, 1:500; catalogue no. ab150074; Abcam, USA) for $1 \mathrm{~h}$ at room temperature. After three washes for $10 \mathrm{~min}$ each with $1 \times \mathrm{PBS}$, the sections were counterstained and mounted with DAPI-Fluoromount-G (catalogue no. 0100-20; SouthernBiotech, AL, USA) and examined under a confocal microscope (A1 R HD25).

To prepare retinal flat-mounts for immunofluorescence analysis, eyeballs were fixed overnight with $2 \%$ paraformaldehyde. Under a dissecting microscope, the neural retina was dissected and immersed in permeabilisation buffer $(2.5 \%$ Triton X-100 and $2.5 \%$ (vol./vol.) Tween-20 in $1 \times$ PBS). The flat-mounts were incubated in permeabilisation buffer on a shaker for 1-4 days at $4^{\circ} \mathrm{C}$, followed by overnight incubation with rabbit anti-IBA-1, goat anti-human collagen IV (1:100, catalogue no. 134001; BioRad, CA, USA), and mouse anti-albumin (1:100, catalogue no. 66051; Proteintech, China) antibodies. After three washes with $1 \times$ PBS for $15 \mathrm{~min}$ each, the retina was incubated for $2 \mathrm{~h}$ in the dark with secondary antibody or fluorescent probe, i.e. donkey anti-rabbit IgG H\&L (Alexa Fluor 488), donkey anti-goat IgG H\&L (Alexa Fluor 555), donkey anti-mouse IgG H\&L (Alexa Fluor 647) (Abcam), or isolectin B4 (IB4; 1:2000, catalogue no. I21411/I32450; Invitrogen) [25]. Flat-mounts were washed three times for 15 min each with $1 \times$ PBS, and mounted with coverslips using Fluoromount-G (catalogue no. 0100-01; SouthernBiotech) followed by examination under a confocal microscope.
Western blotting Cells and retinal tissue samples were lysed in radioimmunoprecipitation assay lysis buffer (catalogue no. 20101ES60; Yeasen, Shanghai, China) containing a complete protease inhibitor cocktail (1:50; Roche, Basel, Switzerland) and PhosStop phosphatase inhibitors (1:10; Roche). The samples were sonicated for $10 \mathrm{~s}$ and then placed on ice for $30 \mathrm{~min}$. Protein concentration was measured with the Pierce bicinchoninic acid protein assay kit (catalogue no. 23225; Thermo Fisher Scientific, MA, USA). After denaturation at high temperature $\left(100^{\circ} \mathrm{C}\right.$ for $\left.5 \mathrm{~min}\right)$, proteins were separated by SDSPAGE and probed with primary and corresponding secondary antibodies (ESM Table 2). Protein bands were detected by chemiluminescence or imaged with an Odyssey infrared imaging system (LI-COR Biosciences, Lincoln, NE, USA).

Microglial quantification and 3D image reconstruction IBA1-positive cells in retinal flat-mount preparations were imaged by confocal microscopy, and the images were transformed into binary and skeleton pictures using ImageJ software (Version 1.52v; NIH, Bethesda, MD, USA). The number of microglia as well as other variables, such as the length of branches or processes and the number of branch points, were quantified according to a previous study [26].

For 3D reconstruction of flat-mounts, the confocal images were processed using IMARIS software (Version 7.4.2; Bitplane/Andor Technology, Belfast, UK) as recommended by the manufacturer.

Retinal vascular leakage examination To detect retinal vascular leakage in Sprague Dawley rats, colour fundus photography and fundus fluorescein angiography were performed at 8 weeks after diabetes onset with or without EPO treatment. In addition, FITC-dextran (catalogue no. FD10S, Sigma, USA) was also utilised to confirm iBRB breakdown in Sprague Dawley rat retinas under different treatments (see ESM for further details).

Flow cytometry analysis and phagocytosis assay $\mathrm{BV}_{2}$ cells were costained with Annexin $\mathrm{V}$ and propidium iodide (PI, catalogue no. 40305ES20; Yeasen). After three washes, the cells were assayed by FACS, and the ratio of apoptotic/necrotic cells to normal cells was calculated accordingly. The phagocytosis assay was performed by calculating the ratio of cells with fluorescent microsphere to total cells as above.

Cell smear immunostaining for phagocytosis assay Phagocytosis by microglia was evaluated with FluoSpheres carboxylatemodified microspheres $(1.0 \mu \mathrm{m}$, Nile Red fluorescent stain [535/575], catalogue no. F8819; Invitrogen) according to the manufacturer's instructions. Briefly, $\mathrm{BV}_{2}$ cells were seeded on coverslips in a 24 -well culture dish $\left(1 \times 10^{4}\right.$ cells/dish). The microspheres were diluted with cell culture medium to a final concentration of $5 \times 10^{7}$ beads per well 
and added to the cells for $2 \mathrm{~h}$ under hypoxic conditions. Groups of cells were also treated with EPO or left untreated for $24 \mathrm{~h}$ under hypoxia.

To perform the phagocytosis assay, $\mathrm{BV}_{2}$ cells in suspension were collected, fixed and permeabilised in tubes containing $1 \times$ PBS with $0.5 \%$ Triton X-100 for 30 min. After blocking with $1 \times$ PBS containing $1 \%$ BSA and $0.2 \%$ Triton $\mathrm{X}-100$ for $1 \mathrm{~h}$, the cells were stained with phalloidin (1:1000, catalogue no. 40736ES750; Yeasen) for $2 \mathrm{~h}$ in the dark and DAPI (catalogue no. 40728ES03; Yeasen) for $10 \mathrm{~min}$, followed by three rinses with $1 \times$ PBS. The cells were mounted on coverslips and stored at room temperature until examination under a confocal microscope. Cells that had engulfed $\geq 2$ microspheres were selected as phagocytic cells, and the ratio of phagocytic cells to total cells was calculated.

ELISA The protein level of vascular endothelial growth factor (VEGF) in the supernatant of $\mathrm{BV}_{2}$ cells under normoxic or hypoxic conditions was measured using an ELISA kit (catalogue no. MMV00, R\&D Systems, Minneapolis, MN, USA). Following the manufacturer's instructions, the amount of VEGF in the samples was determined by using a microplate reader set to $450 \mathrm{~nm}$, which was interpolated from a standard curve constructed from standards after correction for sample dilution.

Statistical analysis Data are expressed as mean \pm SEM and were analysed with the two-tailed Student's $t$ test (unpaired $t$ test) or one-way ANOVA by using IBM SPSS Statistics (version 20; IBM, USA) and GraphPad Prism 8 (GraphPad Software, USA). A $p$ value of $<0.05$ was considered statistically significant.

\section{Results}

EPO inhibits microglial activation in diabetic retinopathy To detect changes in microglial morphology in diabetic retinopathy, we performed immunolabelling of microglial marker IBA-1 and endothelial cell marker IB4 in retinal flat-mounts. In the normal control, IBA-1-positive microglia were evenly distributed and had a ramified morphology (Fig. 1a). However, microglia in diabetic retina had larger cell bodies and an amoeboid morphology, which became more pronounced with diabetes progression. EPO treatment restored the normal morphology of microglia.

To further characterise microglial activation, we analysed the number of branches, branch points, endpoints and branch length of microglia in rat retinas 8 weeks after diabetes onset. Compared with the normal control, in diabetic rats the number of microglia was increased by $76.7 \%(p=0.001)$, whereas branch length, number of branch points and endpoints were decreased by $28.3 \%(p=0.018), 34.6 \%(p=0.005)$ and
45.6\% ( $n=3$ retinas from 3 rats, $p=0.001$ ), respectively. EPO treatment prevented these changes, decreasing the cell number by $27.1 \%(p=0.004)$ compared with that in diabetic retinas without EPO, and increasing branch length by $35.5 \%$ $(p=0.030)$, and the number of branch points and endpoints by $49.4 \%(p=0.003)$ and $67.3 \%(p=0.001)$, respectively (Fig. $1 \mathrm{~b}-\mathrm{f})$. There was no significant difference among the three groups at 2 weeks after diabetes onset (ESM Fig. 1).

We also examined the expression of IBA-1 by western blotting and found that, compared with normal control, the IBA-1 protein level in diabetic retinas was almost unchanged 2 weeks after diabetes onset (Fig. 1g), but there was a nonsignificant increase at 8 weeks $(p=0.150$; Fig. $1 \mathrm{~h})$. The immunofluorescence and western blotting results suggest that IBA-1 immunolabelling is a sensitive method for detecting activated microglia. Based on these observations, we used rat retinas 8 weeks after diabetes onset (referred to as 8week-diabetic rat retinas) for subsequent analyses.

EPO prevents vascular leakage by inhibiting endothelial cell phagocytosis by microglia in the retina in diabetes To assess the correlation between microglial activation and iBRB breakdown, we performed double immunolabelling of IBA-1 and albumin with IB4 in 8-week-diabetic rat retinas with or without EPO treatment. Albumin leakage into the retinal parenchyma was clearly observed in the diabetic retinas compared with the control; this was largely abolished by EPO (Fig. 2a). Three-dimensional reconstruction of retinal flat-mounts confirmed these results (Fig. 2b). To further confirm the iBRB breakdown, a FITC-dextran permeability assay was performed, and the result showed that, compared with the normal control, the leakage of FITC-dextran was increased in diabetic rat retinas, which was prevented by EPO (ESM Fig. 2). In addition, in diabetic retinas, there was more albumin scattered between blood vessels and fewer microglia, and there were a greater number of microglia with amoeboid morphology in close contact with retinal vascular endothelial cells and less albumin (Fig. 2, ESM Video 1), suggesting that activated microglia are effectors in iBRB disruption and cause albumin leakage from blood vessels rather than scavenging the leaked albumin.

To distinguish between these two possibilities, we performed double immunolabelling of retinas using antibodies against IBA-1 and collagen IV (a blood vessel basement membrane marker). The number of ACs and microglia in close contact with endothelial cells in diabetic retinas was $426.5 \%(p=0.001)$ and $85.3 \%$ higher $(p<0.001)$ than in normal control retinas, respectively (Fig. 3a-c, ESM Video 2). EPO administration decreased the number of ACs and the wrapping microglia by $46.7 \%(p=0.011)$ and $23.8 \%$ $(p=0.014)$, respectively. Three-dimensional reconstruction confirmed close contact between activated microglia and endothelial cells in the diabetic retinas, and endothelial cell 
Fig. 1 EPO inhibits microglial activation in rat retina 8 weeks after induction of diabetes. (a) Morphological changes in microglia immunolabelled for IBA-1 (red) and IB4 (green) in normal control and 8-weekdiabetic rat retinas with or without EPO treatment. (b-f)

Quantification of morphological changes in microglia in 8-week rat retinas. Representative binary and skeletonised images are shown (b). (c-f) Summary data of number of microglia/field (c), branch length/cell (d), number of branch points/cell (e) and number of endpoints/cell (f). Data are expressed as mean $\pm \operatorname{SEM}(n=3$ retinas from distinct rats). $(\mathbf{g}, \mathbf{h})$ IBA-1 expression was nonsignificantly increased in 8-weekdiabetic retinas (h; $p=0.150)$, but no changes were observed at 2 weeks (g). Data are expressed as mean \pm SEM (g: $n=6$ retinas; $\mathbf{h}$ : $n=5$ retinas). D, diabetes group; $\mathrm{D}+\mathrm{E}$, diabetes with $\mathrm{EPO}$ treatment group; $\mathrm{N}$, normal control group; $\mathrm{NC}$, negative control. ${ }^{*} p<0.05, * * p<0.01$ by one-way ANOVA. Scale bar, $50 \mu \mathrm{m}$ a
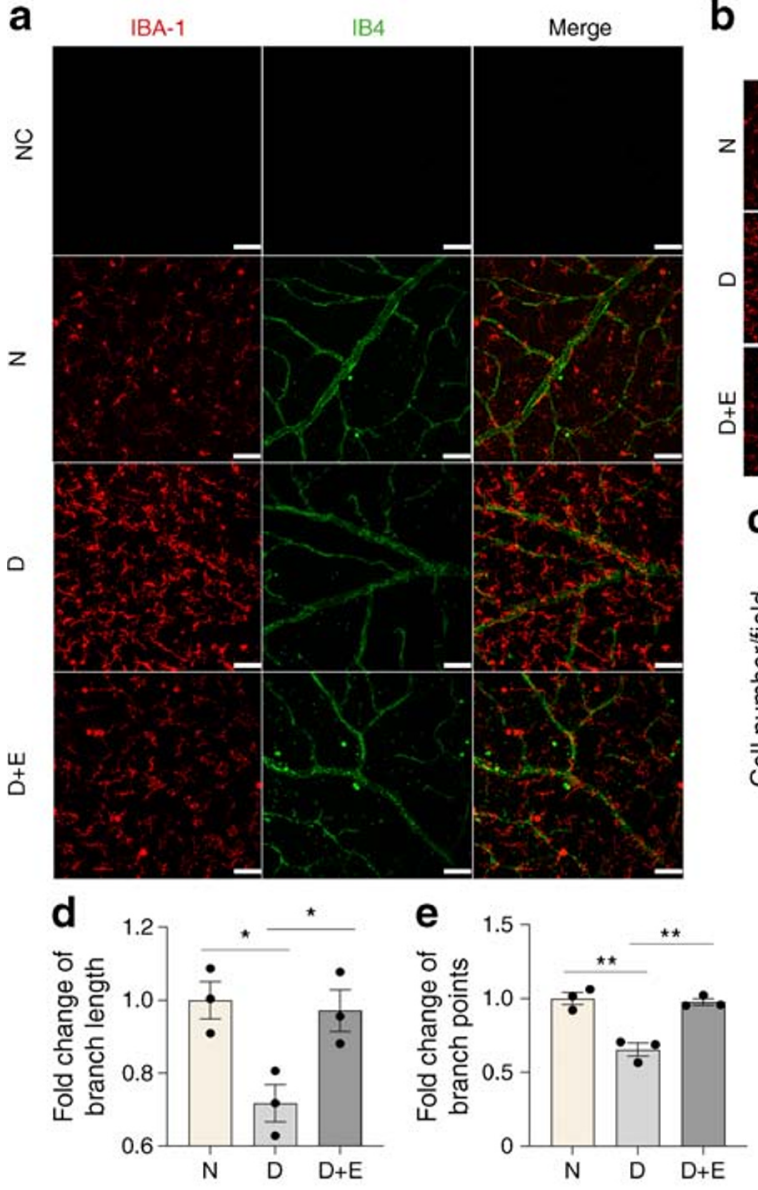

b

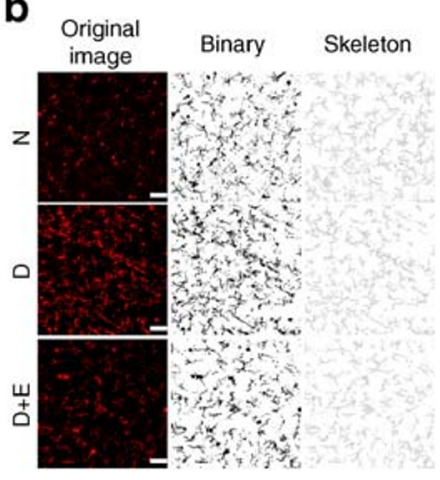

C
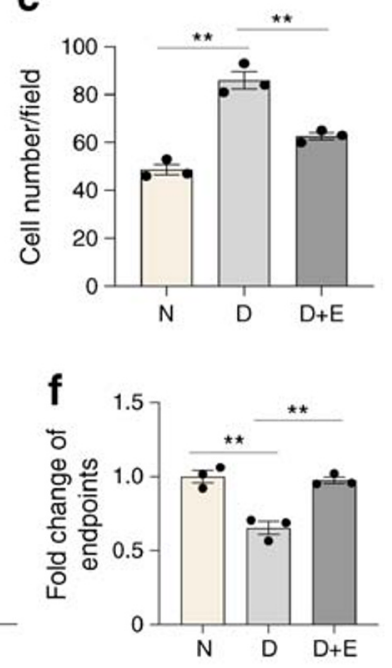
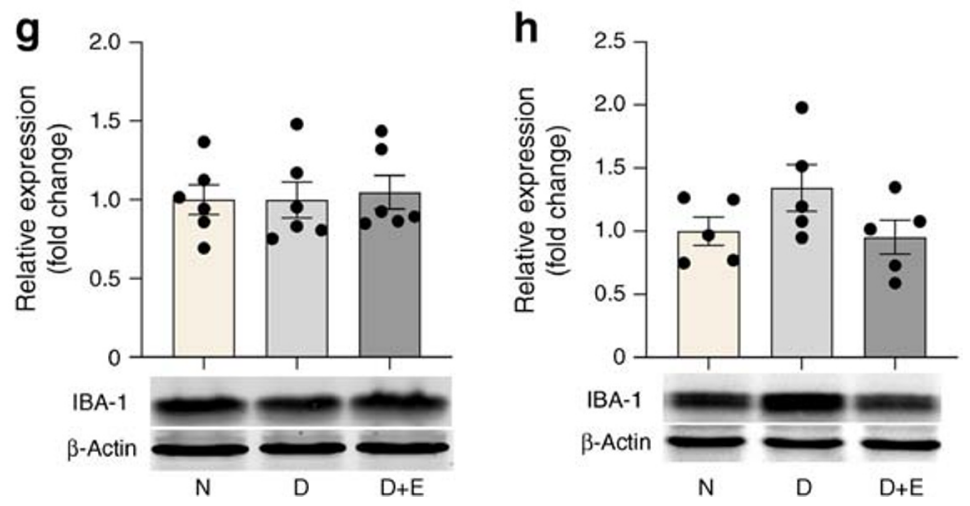

fragments inside microglia implied that the former had been engulfed by the latter (Fig. 3d, ESM Video 3).

\section{EPO inhibits microglial activation under hypoxic conditions}

The in vivo data showed that activated microglia in diabetic retinas engulfed endothelial cells, leading to increased ACs and vascular leakage, while EPO reversed this (Figs 1-3). To mimic the activation of microglia in diabetic retinopathy, we cultured $\mathrm{BV}_{2}$ cells under hypoxic conditions $\left(1 \% \mathrm{O}_{2}\right)$. After $24 \mathrm{~h}$, some cells with an amoeboid form spontaneously detached from the culture dish, while those that remained attached had a ramified morphology (Fig. 4a). The cell number remained relatively unchanged over the $24 \mathrm{~h}$ under hypoxic conditions, whereas in the normoxic control the cell number increased with time (Fig. 4b). We also used FACS with Annexin V/PI labelling to count the total number of cells in the suspension and determine the percentage of living vs dead cells (Fig. 4c-e). More cells were detached in hypoxia than in normoxia $\left(5.53 \times 10^{4} / \mathrm{ml}\right.$ vs $1.51 \times 10^{4} / \mathrm{ml}, p<0.001$; Fig. $4 d)$. Although the fraction of living cells was lower in hypoxia than in normoxia ( $46.3 \%$ vs $81.4 \%$ ), the absolute number of living cells was higher $\left(2.56 \times 10^{4} / \mathrm{ml}\right.$ vs $1.23 \times$ 
Fig. 2 EPO prevents albumin leakage in diabetic rat retina by inhibiting microglia activation. (a) Representative confocal micrographs of IBA-1 (red), IB4 (green) and albumin (white) immunolabelling in retinas from normal rats and diabetic rats 8 weeks after diabetic retinopathy induction. Leaked albumin is indicated by white arrowheads, and the white rectangular boxes indicate colocalisation of IBA-1 and IB4 at a site of increased leakage. Merge-1 shows albumin and IB4 immunoreactivity, and Merge-2 shows the merged signals from all three channels ( $n=6$ retinas from distinct rats). Scale bar, $50 \mu \mathrm{m}$. (b) 3D reconstruction of albumin leakage from blood vessels in the retina. Leaked albumin is visible as white spots (white arrowheads) in the three views of the 3D images. Scale bar, $30 \mu \mathrm{m}$. D/ $/ \mathrm{D}^{\prime}$, diabetes group (different views from the same retina); D+E, diabetes with EPO treatment group; N, normal control group

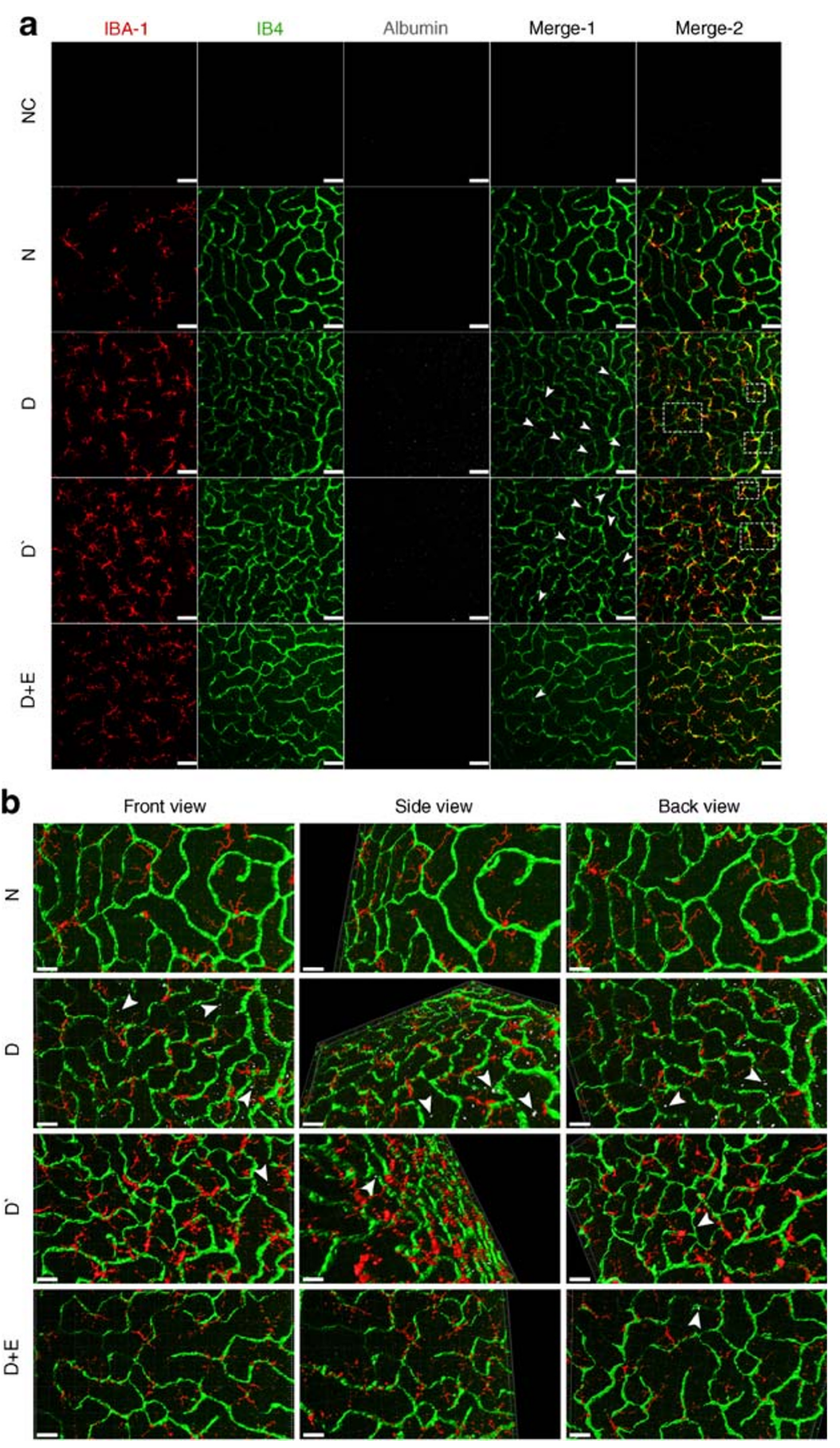

$10^{4} / \mathrm{ml}, p=0.020$; Fig. $4 \mathrm{e}$ ). These results indicate that hypoxia induces microglial activation.

\section{EPO inhibits microglia phagocytosis under hypoxic conditions}

To assess the activation of $\mathrm{BV}_{2}$ cells in suspension, we examined integrin- $\beta 1$ and IBA- 1 levels by western blotting (Fig. $4 \mathrm{f}, \mathrm{g})$. Compared with adherent cells, those that became detached from the culture dish had lower integrin- $\beta 1$ and higher IBA-1 levels, confirming the activation of microglia.
Specifically, relative to the level in adherent control cells, the IBA-1 level in the suspended cells was increased by $373 \%$ $(p=0.040)$ under hypoxic conditions and by $30 \%$ under normoxic conditions $(p=0.284)$.

The phagocytic activity of microglia was examined using fluorescent microspheres. By comparing the number of microglia containing fluorescent microspheres, the phagocytic activity of microglia, indicated by the ratio of the number of phagocytic microglia to the total number of microglia, was 

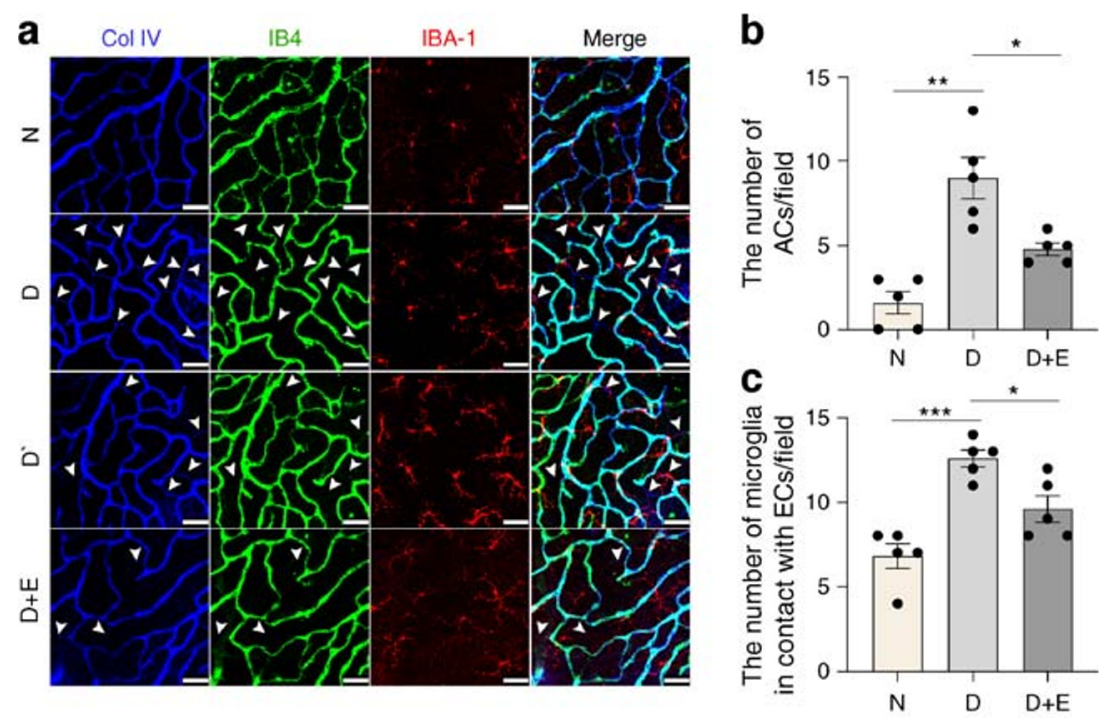

d

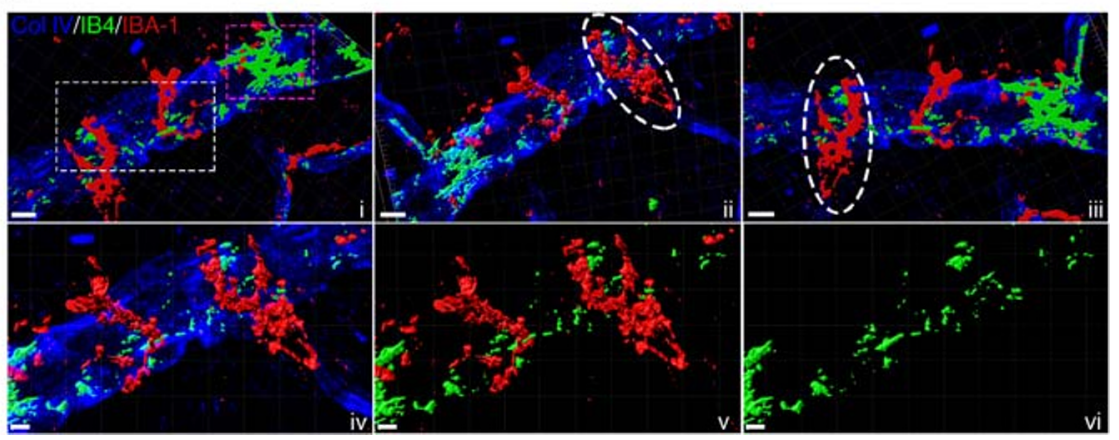

Fig. 3 EPO decreases the number of ACs by inhibiting phagocytosis of endothelial cells by microglia. (a) Representative images of IBA-1 (red), IB4 (green), and collagen IV (Col IV, blue) immunolabelling in retinas from normal rats and diabetic rats 8 weeks after diabetic retinopathy induction. Colocalisation of ACs and IBA-1-positive microglia is indicated by white arrowheads. Scale bar, $50 \mu \mathrm{m}$. (b) Quantification of ACs/ field. (c) Quantification of the number of microglia in close contact with retinal vascular endothelial cells (ECs)/field. Data are expressed as mean \pm SEM ( $n=5$ retinas from distinct rats). (d) Endothelial cells engulfed by activated microglia across the basement membrane of endothelial cells in

$36.2 \%(p=0.031)$ and 93.7\% $(p=0.001)$ higher under normoxic and hypoxic conditions, respectively, in suspended cells compared with that in adherent cells, which was confirmed by FACS (ESM Fig. 3).

To determine the optimal concentration of EPO for modulating microglia activation, $\mathrm{BV}_{2}$ cells cultured subjected to hypoxia were treated with different concentrations of EPO and IBA-1 expression was examined. We found that the IBA-1 level was increased by $50.9 \%(p=0.019)$ under hypoxic conditions compared with normoxic conditions; treatment with $0.5,1$ and $10 \mathrm{U} / \mathrm{ml}$ EPO decreased IBA-1 expression by $38.5 \%(p=0.021), 34.9 \%(p=0.031)$ and $7.8 \%(p=0.749)$, respectively, compared with the level under hypoxic conditions (Fig. 5a). Based on this result, we used an EPO concentration of $1 \mathrm{U} / \mathrm{ml}$ for subsequent experiments. We examined diabetic rat retinas. i-iii show $3 \mathrm{D}$ views of retinal flat-mounts. Endothelial cell fragments $\left(\mathrm{IB}^{+}\right)$are visible inside two activated microglia (white rectangle). A higher number of endothelial cells was associated with fewer activated microglia (purple rectangle). Scale bar, $10 \mu \mathrm{m}$. iv-vi show high-magnification views of endothelial cells phagocytosed by microglia shown in i-iii (white oval/box) (merged channels). Scale bar, $5 \mu \mathrm{m}$. D/D', diabetes group (different views from the same retina); $\mathrm{D}+\mathrm{E}$, diabetes with EPO treatment group; $\mathrm{N}$, normal control group. ${ }^{*} p<0.05$, $* * p<0.01, * * * p<0.001$ by one-way ANOVA

the protein expression of CD11b (a marker of microglial activation) and complement component $1 \mathrm{r}$ (C1r; a marker of phagocytosis) in $\mathrm{BV}_{2}$ cells under hypoxic conditions with or without EPO treatment. Compared with cells under normoxic conditions, CD11b and C1r expression was increased by $170.0 \%(p=0.012)$ and $135.5 \%(p=0.007)$, respectively; EPO treatment decreased the level by $41.3 \%(p=0.042)$ and $35.9 \%$ ( $p=0.049$ ), respectively, relative to that under hypoxic conditions (Fig. 5b,c).

The phagocytic activity of cultured $\mathrm{BV}_{2}$ cells with or without EPO treatment was also evaluated with the fluorescent microsphere assay. Compared with normoxia, the number of cells containing fluorescent microspheres was increased by $118.1 \%$ in hypoxia (vs normoxia, $p<0.001$ ) and decreased by $32.1 \%$ (vs hypoxia, $p=0.005$ ) by EPO treatment (Fig. 


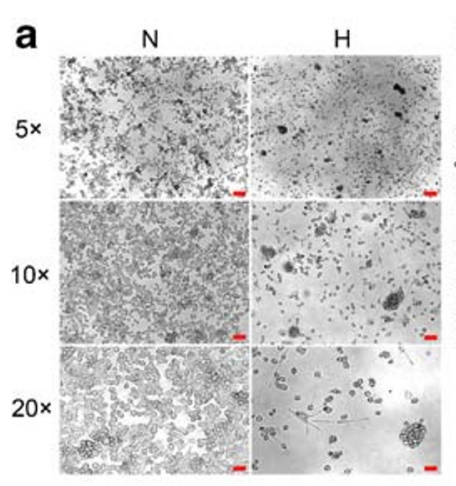

b
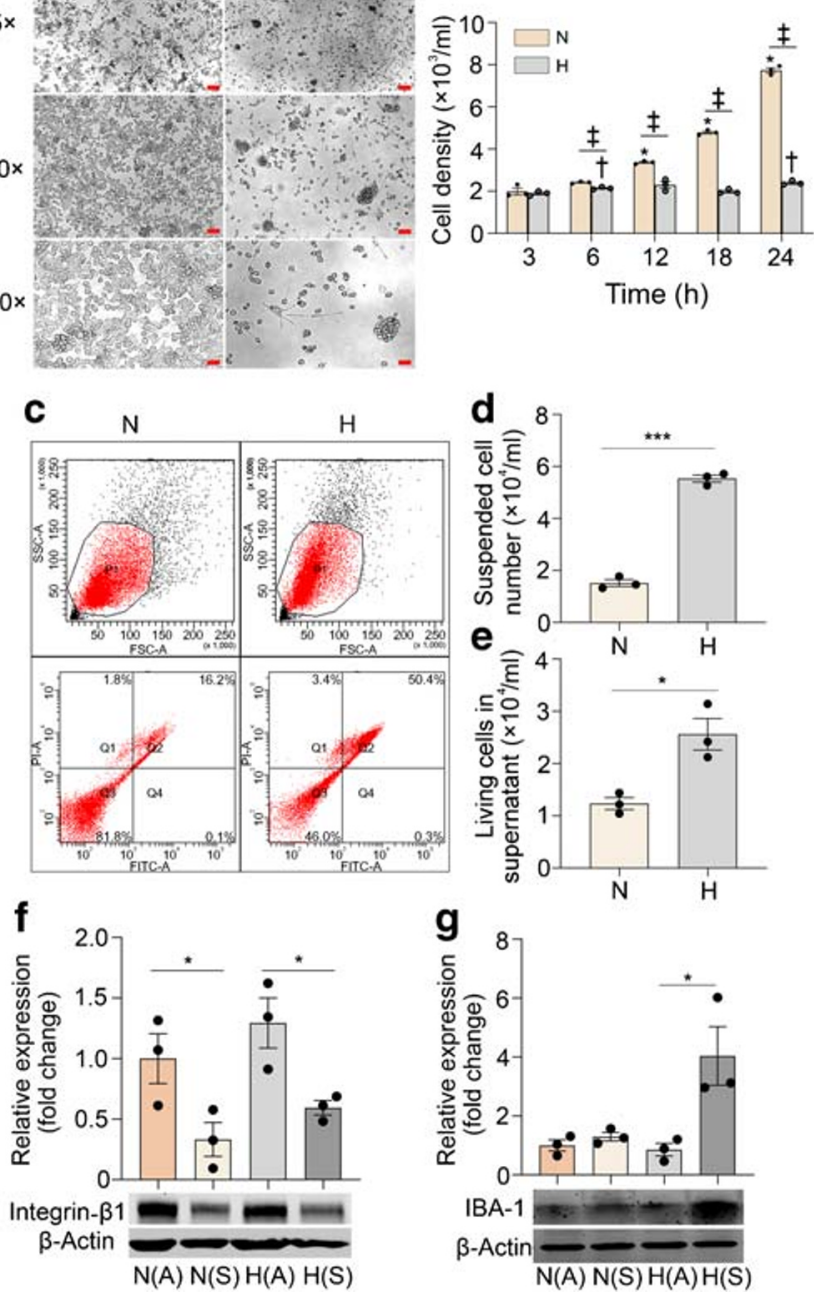

Fig. 4 Hypoxia induces microglia activation. (a) Morphology of $\mathrm{BV}_{2}$ cells after $24 \mathrm{~h}$ in normoxia and hypoxia $\left(1 \% \mathrm{O}_{2}\right)$. Scale bars, 80,40 , $20 \mu \mathrm{m}$ (from top to bottom). (b) Changes in the density of $\mathrm{BV}_{2}$ cells at different time points under normoxic and hypoxic conditions $* p<0.05$ vs $3 \mathrm{~h}$ under normoxic conditions; ${ }^{\dagger} p<0.05$ vs $3 \mathrm{~h}$ under hypoxia; ${ }^{*} p<0.05$ hypoxia vs corresponding time point under normoxia. (c-e) FACS analysis of viable cells under hypoxic conditions. (f) Decreased integrin- $\beta 1$ expression in suspended cells compared with adherent cells under normoxic and hypoxic conditions. (g) Increased IBA-1 protein level in suspended cells compared with adherent cells in hypoxia. Data are expressed as mean \pm SEM ( $n=3$ from separate cell samples). H, hypoxia group; $\mathrm{H}(\mathrm{A})$, adherent cells under hypoxic conditions; $\mathrm{H}(\mathrm{S})$, suspended (detached) cells under hypoxic conditions; N, normoxia group; N(A), adherent cells under normoxic conditions; N(S), suspended (detached) cells under normoxic conditions. The data were analysed by one-way ANOVA (f, g) and unpaired $t$ test $(\mathbf{b}, \mathbf{d}, \mathbf{e}) ;(\mathbf{d}-\mathbf{g}) * p<0.05, * * * p<0.001$

5d,e). Similarly, F-actin immunoreactivity, as indicated by phalloidin staining, was apparently increased under hypoxia, which was slightly decreased by EPO (Fig. 5e).

We also used primary microglia to confirm the above changes. Compared with the microglia under normoxic conditions, the cellular bodies of primary microglia were enlarged slightly (ESM Fig. 4a). In addition, protein levels of IBA-1, CD11b and C1r were increased under hypoxic conditions, and this increase was reduced by EPO (ESM Fig. 4b-e). In terms of phagocytic activity, the ratio of primary microglia containing fluorescent microspheres to total microglia was increased by $19.9 \%(p=0.007)$ under hypoxic conditions, but the level under hypoxic conditions was decreased by $18.1 \%$ ( $p=0.005$ ) by EPO (ESM Fig. 5).

EPO blocks endothelial cell phagocytosis by microglia under hypoxic conditions Our results showed that the phagocytic activity of microglia was enhanced under diabetic and hypoxic conditions (Figs 3 and 5). To determine whether activated microglia directly engage endothelial cells we used a $\mathrm{BV}_{2}$ cell/HRMEC coculture system to mimic their interaction and labelled the cells with antibodies against CD11b and CD31 (an endothelial cell marker) (Fig. 6a).

In the absence of microglia, HRMEC morphology did not differ between normoxic and hypoxic conditions. When cocultured with microglia, the migration of microglia to the opposite side of the transwell, which was coated with fibronectin and seeded HRMECs, was not detected in normoxia, but it was enhanced in hypoxia, as evidenced by the disruption of the HRMEC monolayer and phagocytosis of these cells by migrated microglia. EPO treatment abrogated this effect (Fig. 6b). The above changes were also recapitulated in an RM/HRMEC coculture (ESM Fig. 6).

EPO inhibits microglia phagocytosis via activation of Src/Akt/ cofilin signalling $\mathrm{Src} / \mathrm{Akt} /$ cofilin signalling mediates cellular cytoskeletal remodelling in phagocytosis [27-29]. We therefore investigated the effect of EPO on the activation of $\mathrm{Src} /$ Akt/cofilin signalling in $\mathrm{BV}_{2}$ cells. Both phosphorylated and total protein levels of Src, Akt and cofilin were downregulated in hypoxic vs normoxic conditions (Fig. 7). We calculated the ratio of phosphorylated protein to actin in order to detect changes in the Src/Akt/cofilin pathway and found that, compared with the normal control, the ratios of phosphorylated (p-)Src, p-Akt and p-cofilin to actin were decreased under hypoxic conditions by $74.2 \%$ ( $p=0.022), 47.8 \%(p=0.038)$, and $39.7 \%(p=0.043)$, respectively. On the other hand, EPO treatment increased p-Src/actin, p-Akt/actin, and p-cofilin/ actin ratios by $108.4 \%(p=0.047), 83.1 \%(p=0.049)$, and $62.2 \%(p=0.040)$, respectively, relative to cells under hypoxic conditions (Fig. 7b-d). The ratios of the phosphorylated protein/total protein and total protein/actin are also presented in ESM Fig. 7.

To further clarify a role for Src/Akt/cofilin signalling in mediating the effects of $\mathrm{EPO}$ under hypoxic conditions, $\mathrm{BV}_{2}$ cells were treated with activators of Src and Akt and the expression of downstream effectors was evaluated. Src family 
Fig. 5 EPO inhibits activation and suppresses phagocytic activity in microglia under hypoxic conditions. (a)

Expression of IBA- 1 in $\mathrm{BV}_{2}$ cells under hypoxic conditions treated with different concentrations of EPO ( $n=3$ from separate cell samples). (b, c) Expression of CD11b (b, $n=4$ from separate cell samples) and C1r (c, $n=4$ from separate cell samples) proteins in $\mathrm{BV}_{2}$ cells under hypoxic conditions with or without EPO treatment $(1 \mathrm{U} / \mathrm{ml})$. (d)

Quantification of phagocytic cells ( $n=6$ from separate cell samples).

(e) Phagocytosis assay for

microglia under normoxia and hypoxia with or without EPO, MK2206, Src family activator and SC79 treatment. The column labelled 'Magnification' shows a high-magnification view of the microglia marked by a rectangle in the merged photos immediately to the left. Data are expressed as mean \pm SEM. Scale bar, $25 \mu \mathrm{m}$. $\mathrm{H}$, hypoxia group; $\mathrm{H}+\mathrm{E}$, hypoxia+EPO treatment group; $\mathrm{N}$, normoxia group. $* p<0.05$, $* * p<0.01, * * * p<0.001$ by oneway ANOVA
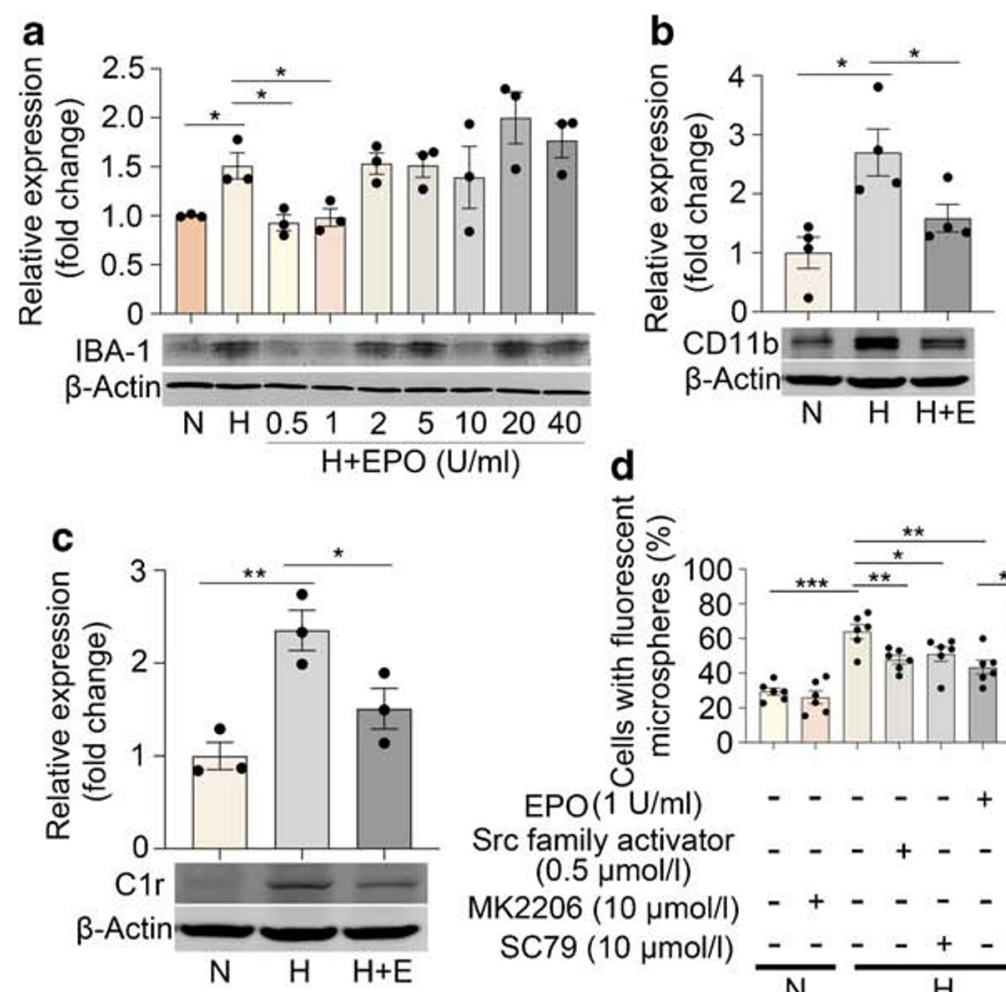

d
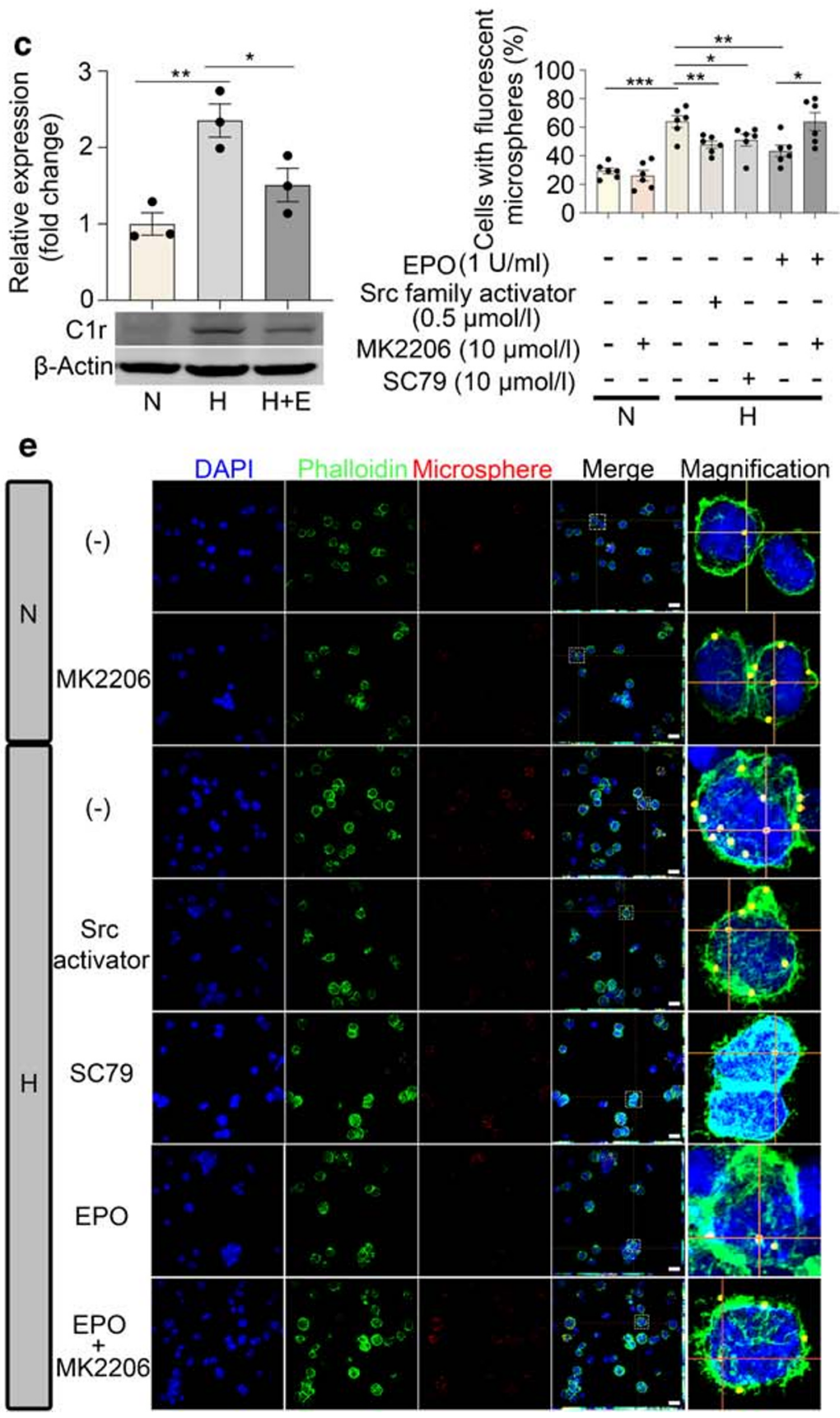
Fig. 6 EPO prevents endothelial cell phagocytosis by microglia under hypoxia. (a) Schematic illustration of the coculture system. (b) Interaction of microglia and endothelial cells (ECs) revealed by $\mathrm{CD} 11 \mathrm{~b}$ and CD31 immunolabelling ( $n=6$ from separate cell samples). Scale bar, $25 \mu \mathrm{m}$. ECM, endothelial cell medium; $\mathrm{H}$, hypoxia group; $\mathrm{HG}$, high-glucose medium; $\mathrm{N}$, normoxia group a

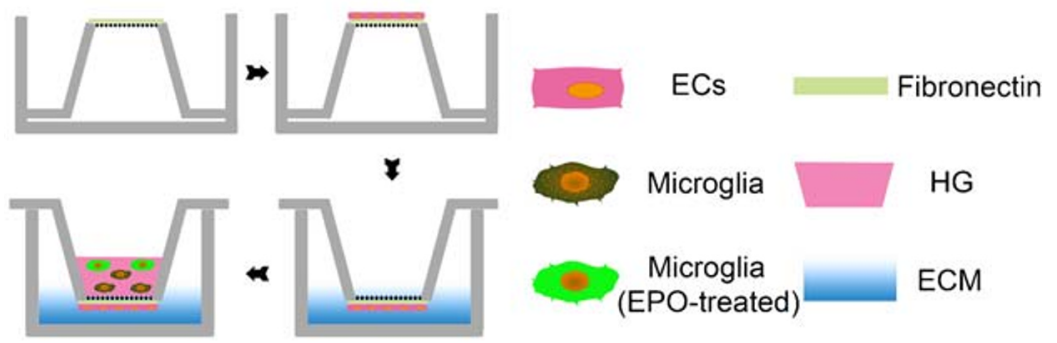

b

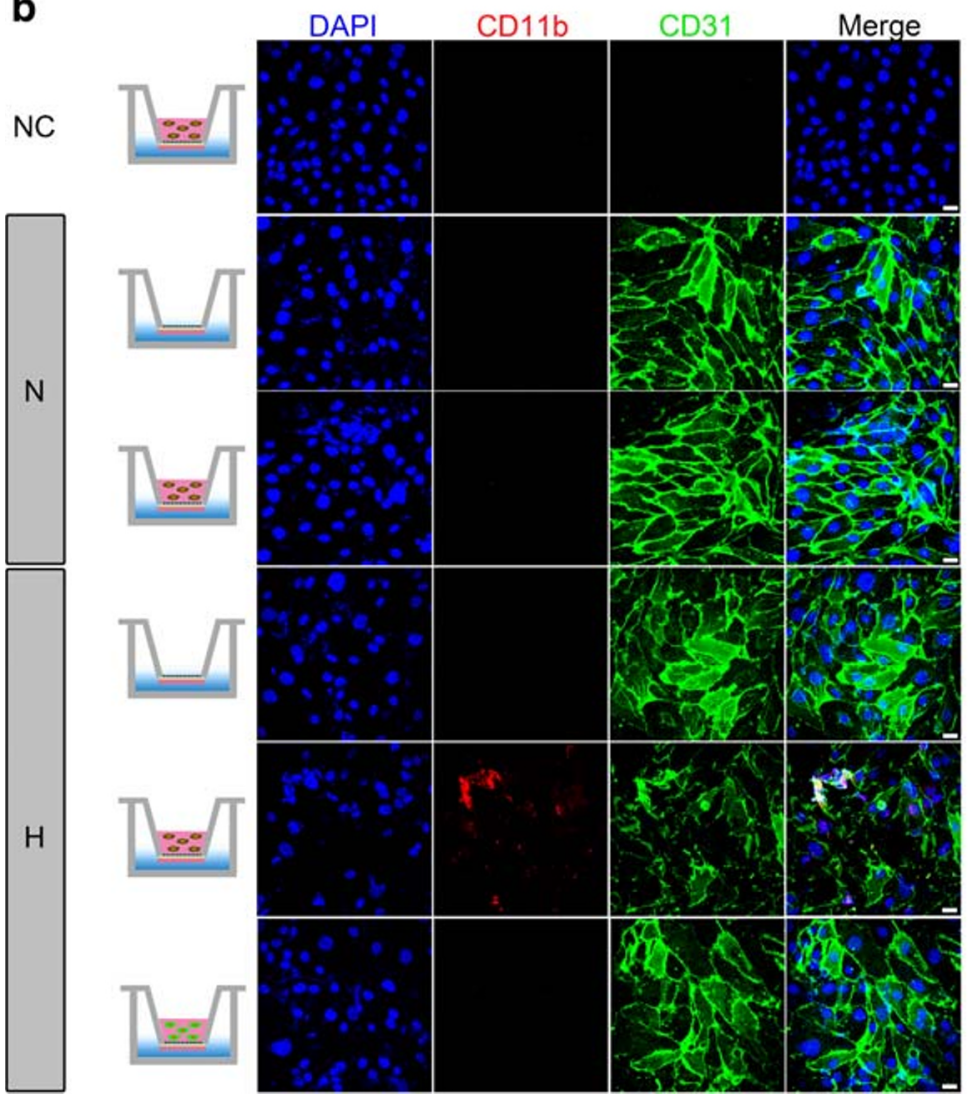

activator increased $\mathrm{p}$-Akt/actin and $\mathrm{p}$-cofilin/actin ratios by $116.5 \%(p=0.009)$ and $244.8 \%(p=0.018)$, respectively, compared with untreated cells under hypoxic conditions. The Akt activator SC79 increased p-cofilin/actin by $211.5 \%$ $(p=0.043)$ but had no effect on $\mathrm{p}$-Src expression $(64.3 \%$ increase, $p=0.100$ ) (Fig. 7). These results demonstrate that the Src/Akt/cofilin signalling pathway is involved in the phagocytic activity of microglia.

We used the fluorescent microsphere assay to confirm this finding. Like EPO, Src and Akt activators inhibited the phagocytic activity of microglia under hypoxic conditions by $25.4 \%$ $(p=0.007)$ and $20.1 \%(p=0.043)$, respectively (Fig. $5 \mathrm{~d}, \mathrm{e})$. The effect of EPO was abolished by the Akt inhibitor MK2206, which increased phagocytosis in EPO-treated cells by $147.2 \%(p=0.021)$. These data indicate that the modulatory effect of EPO on microglial phagocytic activity in diabetic retinopathy is exerted through activation of Src/Akt/ cofilin signalling.

\section{Discussion}

Microglial activation in diabetic retinopathy has been widely studied and has been shown to precede the formation of vascular lesions. Microglia can be activated by various factors, including Amadori-glycated albumin and damaged cells. Activated microglia are characterised by enhanced migratory and phagocytic capacities and regulate the microenvironment through the release of cytokines [11-14]; however, they can also perturb homeostasis in the microenvironment and cause damage to neurons or vascular cells. Excessive cytokine secretion and enhanced phagocytosis by microglia $[13,14]$ 
Fig. 7 EPO inhibits phagocytosis of $\mathrm{BV}_{2}$ cells through activation of the $\mathrm{Src} / \mathrm{Akt} / \mathrm{cofilin}$ pathway in hypoxia. (a) Phosphorylated and total protein levels of Src, Akt and cofilin under different treatments. (b-d) Ratios of phosphorylated protein to $\beta$-actin. Data are expressed as mean $\pm \operatorname{SEM}(n=3$ from separate cell samples). $\mathrm{H}$, hypoxia; MK, MK2206 treatment; N, normoxia; SFA, Src family activator treatment. $* p<0.05, * * p<0.01$, by one-way ANOVA
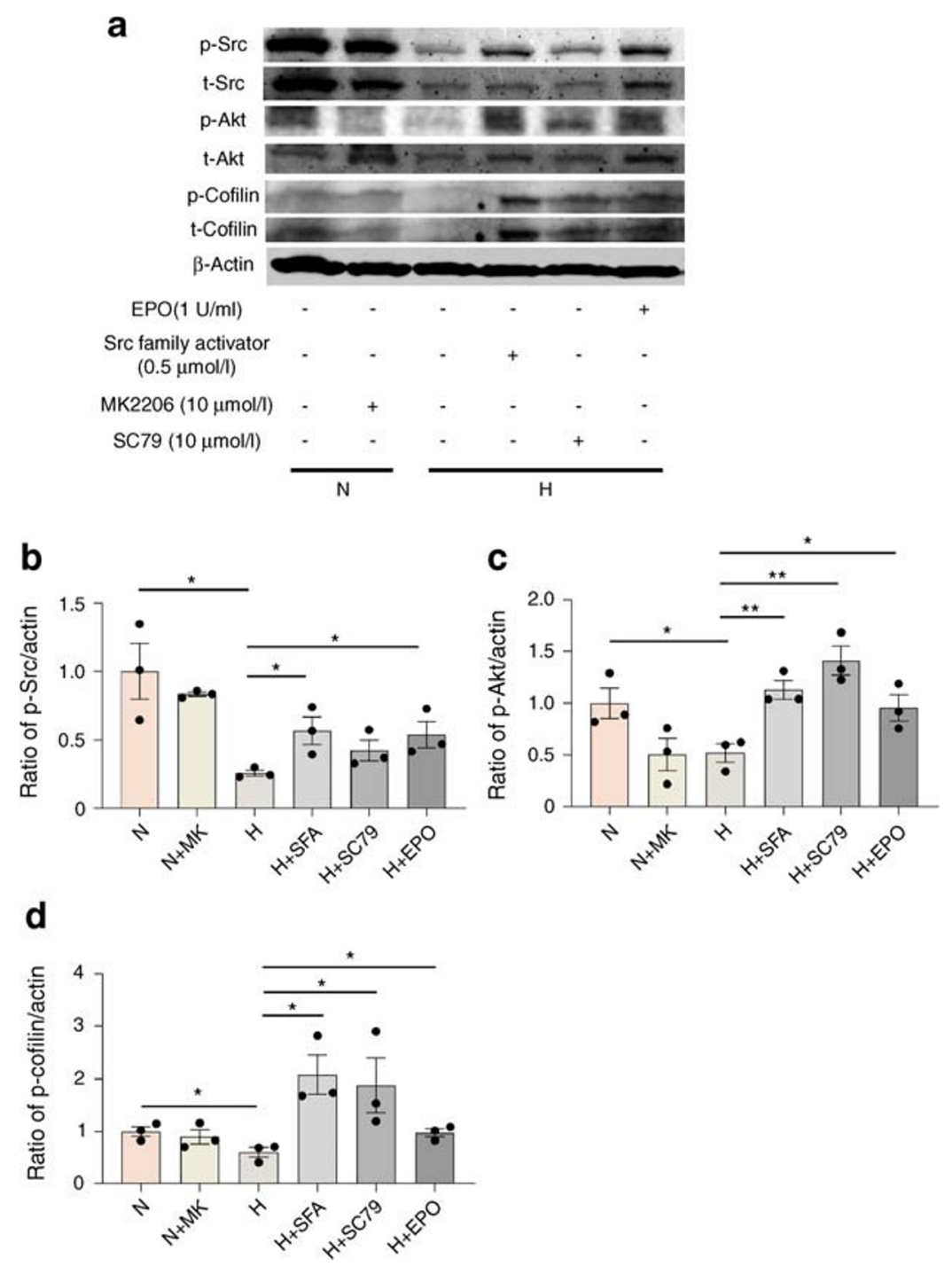

have been implicated in the pathogenesis of diseases such as retinitis pigmentosa [30]. Activated microglia accumulate in areas of vascular leakage [15] and participate in iBRB disruption through the inflammatory response, which enhances leakage from blood vessels [14]. Suppressing inflammation induced by microglia has been shown to have a prophylactic effect against diabetic retinopathy $[31,32]$. Moreover, coculture of microglia with R28 (an immortalised rat retinal precursor cell line) resulted in the production of neurotoxins [33]. Collectively, these findings suggest a detrimental role for activated microglia in diabetic retinopathy.

iBRB breakdown is an early event in the pathogenesis of diabetic retinopathy that involves the apoptosis of endothelial cells and pericytes [34-36] and a decrease in tight junction proteins between endothelial cells $[37,38]$. In addition, VEGF and fibroblast growth factor levels induce leakage from blood vessels. Decreased production of neurotrophic factors by Müller cells, astrocytes and neurons as well as oxidative stress also contribute to iBRB breakdown $[34,35,39]$. However, few studies have examined the role of microglia in iBRB breakdown, although one report demonstrated an indirect interaction in a mouse model [40].

In this study, we observed that microglia activation in diabetic retinopathy was associated with morphological changes, i.e. ramified microglia assumed an amoeboid form (Fig. 1). These microglia could engulf endothelial cells by penetrating the basement membrane of blood vessels, leading to iBRB disruption, since we found endothelial cell fragments inside activated microglia in diabetic retinas (Fig. 3c). In addition, the increased number of ACs and aggravation of vascular leakage further contributed to diabetic retinopathy progression (Figs 2 and 3a), consistent with previously published findings $[15,41]$.

EPO was previously found to exert protective effects in diabetic retinopathy [19-22]. In our previous study we demonstrated that EPO preserved the integrity of the BBRB and attenuated vascular leakage through inhibition of hypoxia-inducible factor- $1 \alpha /$ VEGF signalling [19]. In addition, EPO prevented 
retinal microvascular damage by reducing pericyte loss, suppressing oxidative stress and the formation of advanced glycation end-products, etc. [42]. Here, we showed that EPO blocked the phagocytosis of endothelial cells in diabetic retinopathy by inhibiting microglial activation.

Given that hypoxia is a feature of diabetic retinopathy pathogenesis [43], we cultured microglia under hypoxic conditions to induce their activation. The dimorphism of microglia under these conditions, i.e. adherent cells with ramified morphology and cluster-forming suspended (detached) cells with a round and amoeboid form (Fig. 4a), has been previously described [44]. The latter cells showed enhanced migration and phagocytosis, which is consistent with our in vivo findings (Figs 4f, 5c-e and ESM Figs 3 and 5). Based on our observation that activated microglia engulfed endothelial cells, we performed in vitro experiments to determine whether there is direct interaction between them. In the coculture system, activated microglia migrated to the opposite side of the transwell membrane and engulfed endothelial cells, causing the disruption of the cell monolayer (Fig. 6, ESM Fig. 6). In an earlier study, vascular leakage was reduced in mouse retina by inhibiting colony-stimulating factor 1 receptor via suppression of inflammatory cytokines released by microglia/ macrophages [45]. Under hypoxic conditions, VEGF production was increased by microglia (ESM Fig. 8). The junctional proteins (VE-cadherin and zonula occludens-1 [ZO-1]) in HRMECs remained relatively unchanged in response to hypoxia over a short time $(9 \mathrm{~h})$, while they became disrupted and downregulated under hypoxic conditions over a longer time (24 h) (ESM Figs 9-11). Although we cannot exclude the involvement of hypoxia and microglia-derived inflammatory factors, including VEGF in iBRB breakdown, our data suggest that phagocytosis of endothelial cells by activated microglia plays a central role in this process.

We examined the mechanistic basis for enhanced phagocytosis by microglia under hypoxia. The Src/Akt/cofilin pathway and actin-related protein (Arp2/3) play a critical role in Factin polymerisation; however, no difference in the expression of Arp2/3 was observed under normoxia vs hypoxia in our study (data not shown). The phosphorylation of Src, Akt, and cofilin were decreased under hypoxia, an effect that was prevented by EPO. The activators of Src and Akt, like EPO, alleviated the phagocytic effect of microglia, suggesting a regulatory mechanism by which the phagocytic activity of microglia is enhanced by hypoxia as in diabetic retinopathy.

We observed two distinct phenomena in retinal flat-mount preparations from rats with STZ-induced diabetes. First, there was greater accumulation of activated microglia around vessels that showed obvious leakage (Fig. 2). In addition, more ACs were present at sites harbouring activated microglia (Fig. 3a). We speculate that microglia are activated and internalise endothelial cells in the early stage of diabetic

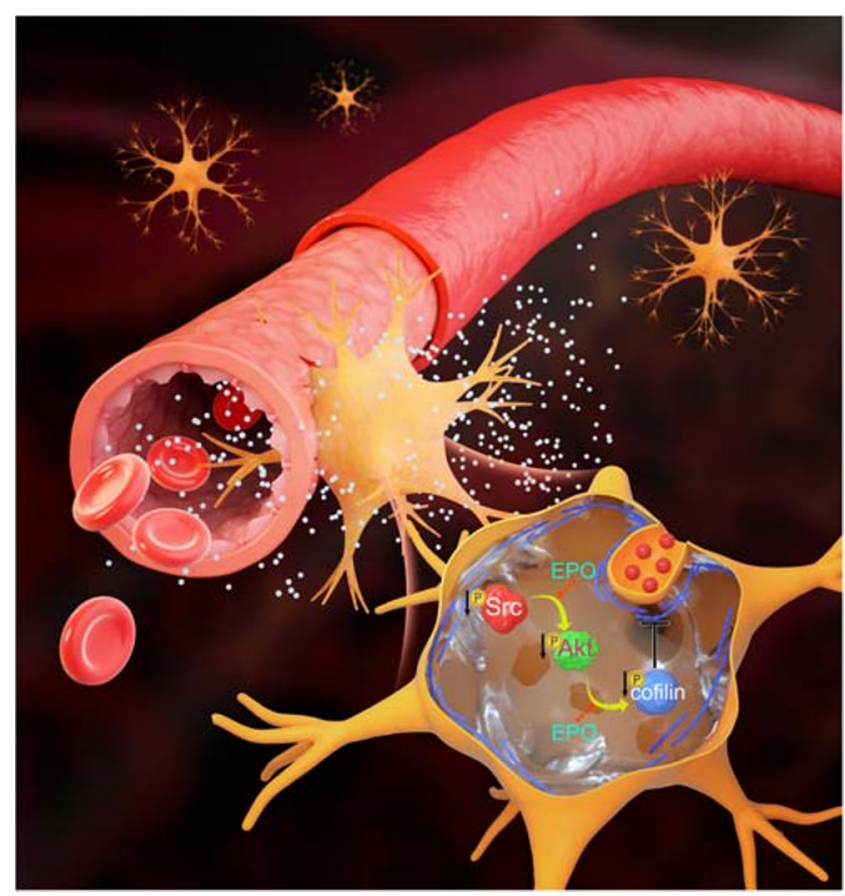

Fig. 8 EPO prevents iBRB damage by activated microglia in diabetic retinopathy via activation of the $\mathrm{Src} / \mathrm{Akt} / \mathrm{cofilin}$ pathway. In diabetic retinopathy or under hypoxic conditions, activated microglia engulf endothelial cells, resulting in iBRB breakdown, an increase in ACs and albumin leakage. These effects are abolished by EPO through activation of $\mathrm{Src} / \mathrm{Akt} / \mathrm{cofilin}$ signalling in microglia. The red beads represent fluorescent microspheres inside microglia

retinopathy and migrate thereafter to other locations for asyet-unknown reasons.

In conclusion, this study provides new insight into early events in the pathogenesis of diabetic retinopathy; specifically, the contribution of activated microglia to iBRB breakdown. Evidence from in vitro and in vivo experiments revealed a direct interaction between microglia and endothelial cells that leads to iBRB disruption. This study is also the first to provide evidence that EPO protects endothelial cells from phagocytosis by activated microglia through activation of the Src/Akt/cofilin signalling pathway, thereby preserving iBRB integrity (Fig. 8). An open question is whether microglial activation is a cause or consequence of endothelial cell death, which could be addressed by examining the correlation between activated microglia and iBRB breakdown in experimental diabetic retinopathy by depleting or inactivating microglia in situ. Whether or not EPO could counteract the neurotoxicity caused by STZ in retina cannot be precluded yet, although the neurotoxicity induced by STZ in retina seems unlikely due to the presence of blood-ocular barrier, similar to the blood-brain barrier [46]. Non-STZ diabetic models should be considered in further studies. Nonetheless, our findings highlight the therapeutic potential of EPO for the treatment of diabetic retinopathy. 
Acknowledgements We acknowledge the support from the Laboratory of Clinical and Vision Science (LCVS; Tongji University School of Medicine, Shanghai, China). We are also grateful to all colleagues, who were involved in this study.

Data availability The datasets generated and/or analysed during the current study are available from the corresponding author on reasonable request.

Funding This work was supported by grants from the National Natural Science Foundation of China $(81570852,81870667,81970810$, 81970811), the Science and Technology Commission of Shanghai Municipality (17ZR1431300, 18411953400, 19495800700), the Shanghai Pujiang Program (15PJ1408700), the National Key Basic Research Program of China (2017YFA0104100, 2016YFA0101302) and Shanghai East Hospital Grant ZJ2014-ZD-002.

Authors' relationships and activities The authors declare that there are no relationships or activities that might bias, or be perceived to bias, their work.

Contribution statement HX, GTX and JFZ contributed to the conception and design of the experiments and were responsible for data collection, analysis and interpretation. CYZ, DDL, QY, LT and TQW contributed to the analysis of in vitro data and article revision. HX and JFZ drafted the article. HBT, LXL, JYX, FRG, JW, CXJ, GXX and WYL contributed to the data analysis and revised the article critically for important intellectual content. All authors approved the final version of the manuscript for submission. JFZ and GTX are guarantors of this work, who have full access to all the data in this study and take responsibility for the integrity and accuracy of the data.

\section{References}

1. Yau JW, Rogers SL, Kawasaki R et al (2012) Global prevalence and major risk factors of diabetic retinopathy. Diabetes Care 35(3): 556-564. https://doi.org/10.2337/dc11-1909

2. Cheung N, Mitchell P, Wong TY (2010) Diabetic retinopathy. Lancet 376(9735):124-136. https://doi.org/10.1016/s01406736(09)62124-3

3. Wong TY, Cheung CM, Larsen M, Sharma S, Simo R (2016) Diabetic retinopathy. Nat Rev Dis Primers 2:16012. https://doi. org/10.1038/nrdp.2016.12

4. Araszkiewicz A, Rogowicz-Frontczak A, Zozulinska-Ziolkiewicz D, Pilacinski S, Wykretowicz A, Wierusz-Wysocka B (2011) Presence of retinopathy in type 1 diabetic patients is associated with subclinical macroangiopathy. Scand J Clin Lab Invest 71(7):563568. https://doi.org/10.3109/00365513.2011.593268

5. Romero-Aroca P, Baget-Bernaldiz M, Reyes-Torres J et al (2012) Relationship between diabetic retinopathy, microalbuminuria and overt nephropathy, and twenty-year incidence follow-up of a sample of type 1 diabetic patients. J Diabetes Complicat 26(6): 506-512. https://doi.org/10.1016/j.jdiacomp.2012.06.010

6. Simo R, Hernandez C, European Consortium for the Early Treatment of Diabetic Retinopathy (EUROCONDOR) (2012) Neurodegeneration is an early event in diabetic retinopathy: therapeutic implications. Br J Ophthalmol 96(10):1285-1290. https:// doi.org/10.1136/bjophthalmol-2012-302005

7. Gaucher D, Chiappore JA, Paques M et al (2007) Microglial changes occur without neural cell death in diabetic retinopathy. Vis Res 47(5):612-623. https://doi.org/10.1016/j.visres.2006.11.017
8. Xu H, Chen M, Mayer EJ, Forrester JV, Dick AD (2007) Turnover of resident retinal microglia in the normal adult mouse. Glia 55(11): 1189-1198. https://doi.org/10.1002/glia.20535

9. Yuan TF, Liang YX, Peng B, Lin B, So KF (2015) Local proliferation is the main source of rod microglia after optic nerve transection. Sci Rep 5:10788. https://doi.org/10.1038/srep10788

10. Karlstetter M, Scholz R, Rutar M, Wong WT, Provis JM, Langmann T (2015) Retinal microglia: just bystander or target for therapy? Prog Retin Eye Res 45:30-57. https://doi.org/10.1016/j. preteyeres.2014.11.004

11. Chen X, Zhou H, Gong Y, Wei S, Zhang M (2015) Early spatiotemporal characterization of microglial activation in the retinas of rats with streptozotocin-induced diabetes. Graefes Arch Clin Exp Ophthalmol 253(4):519-525. https://doi.org/10.1007/s00417-0142727-y

12. Ibrahim AS, El-Remessy AB, Matragoon S et al (2011) Retinal microglial activation and inflammation induced by amadoriglycated albumin in a rat model of diabetes. Diabetes 60(4):11221133. https://doi.org/10.2337/db10-1160

13. Altmann C, Schmidt MHH (2018) The role of microglia in diabetic retinopathy: inflammation, microvasculature defects and neurodegeneration. Int J Mol Sci 19(1):110. https://doi.org/10.3390/ ijms 19010110

14. Grigsby JG, Cardona SM, Pouw CE et al (2014) The role of microglia in diabetic retinopathy. J Ophthalmol 2014:705783. https://doi.org/10.1155/2014/705783

15. Mendiola AS, Garza R, Cardona SM et al (2016) Fractalkine signaling attenuates perivascular clustering of microglia and fibrinogen leakage during systemic inflammation in mouse models of diabetic retinopathy. Front Cell Neurosci 10:303. https://doi.org/10.3389/ fncel.2016.00303

16. Caprara C, Grimm C (2012) From oxygen to erythropoietin: relevance of hypoxia for retinal development, health and disease. Prog Retin Eye Res 31(1):89-119. https://doi.org/10.1016/j.preteyeres. 2011.11.003

17. Zhang J, Wu Y, Jin Y et al (2008) Intravitreal injection of erythropoietin protects both retinal vascular and neuronal cells in early diabetes. Invest Ophthalmol Vis Sci 49(2):732-742. https://doi. org/10.1167/iovs.07-0721

18. Zhang J, Hu LM, Xu G et al (2010) Anti-VEGF effects of intravitreal erythropoietin in early diabetic retinopathy. Front Biosci (Elite Ed) 2:912-927. https://doi.org/10.2741/e151

19. Xu H, Zhang L, Gu L et al (2014) Subretinal delivery of AAV2mediated human erythropoietin gene is protective and safe in experimental diabetic retinopathy. Invest Ophthalmol Vis Sci 55(3): 1519-1530. https://doi.org/10.1167/iovs.13-13155

20. Gu L, Xu H, Wang F et al (2014) Erythropoietin exerts a neuroprotective function against glutamate neurotoxicity in experimental diabetic retina. Invest Ophthalmol Vis Sci 55(12):8208-8222. https://doi.org/10.1167/iovs.14-14435

21. Zhang C, Xie H, Yang Q et al (2019) Erythropoietin protects outer blood-retinal barrier in experimental diabetic retinopathy by upregulating ZO-1 and occludin. Clin Exp Ophthalmol 47(9):11821197. https://doi.org/10.1111/ceo.13619

22. Hu LM, Luo Y, Zhang J et al (2011) EPO reduces reactive gliosis and stimulates neurotrophin expression in Muller cells. Front Biosci (Elite Ed) 3:1541-1555. https://doi.org/10.2741/e355

23. Li W, Sinclair SH, Xu GT (2010) Effects of intravitreal erythropoietin therapy for patients with chronic and progressive diabetic macular edema. Ophthalmic Surg Lasers Imaging 41(1):18-25. https://doi.org/10.3928/15428877-20091230-03

24. Akhtar-Schäfer I, Wang L, Krohne TU, Xu H, Langmann T (2018) Modulation of three key innate immune pathways for the most common retinal degenerative diseases. EMBO Mol Med 10(10). https://doi.org/10.15252/emmm.201708259 
25. Dannhausen K, Rashid K, Langmann T (2018) Microglia analysis in retinal degeneration mouse models. Methods Mol Biol 1753: 159-166. https://doi.org/10.1007/978-1-4939-7720-8_10

26. Young K, Morrison H (2018) Quantifying microglia morphology from photomicrographs of immunohistochemistry prepared tissue using ImageJ. J Vis Exp 136:57648. https://doi.org/10.3791/57648

27. Mylvaganam SM, Grinstein S, Freeman SA (2018) Picket-fences in the plasma membrane: functions in immune cells and phagocytosis. Semin Immunopathol 40(6):605-615. https://doi.org/10.1007/ s00281-018-0705-x

28. Tao H, Yancey PG, Babaev VR et al (2015) Macrophage SR-BI mediates efferocytosis via Src/PI3K/Rac1 signaling and reduces atherosclerotic lesion necrosis. J Lipid Res 56(8):1449-1460. https://doi.org/10.1194/jlr.M056689

29. Lu C, Fan Z, Xing D (2016) Photo-enhancement of macrophage phagocytic activity via Rac1-mediated signaling pathway: Implications for bacterial infection. Int J Biochem Cell Biol 78: 206-216. https://doi.org/10.1016/j.biocel.2016.06.010

30. Zhao L, Zabel MK, Wang X et al (2015) Microglial phagocytosis of living photoreceptors contributes to inherited retinal degeneration. EMBO Mol Med 7(9):1179-1197. https://doi.org/10.15252/ emmm.201505298

31. Zhang T, Ouyang H, Mei X et al (2019) Erianin alleviates diabetic retinopathy by reducing retinal inflammation initiated by microglial cells via inhibiting hyperglycemia-mediated ERK1/2-NF-KB signaling pathway. FASEB J 33(11):11776-11790. https://doi. org/10.1096/fj.201802614RRR

32. McVicar CM, Hamilton R, Colhoun LM et al (2011) Intervention with an erythropoietin-derived peptide protects against neuroglial and vascular degeneration during diabetic retinopathy. Diabetes 60(11):2995-3005. https://doi.org/10.2337/db11-0026

33. Krady JK, Basu A, Allen CM et al (2005) Minocycline reduces proinflammatory cytokine expression, microglial activation, and caspase-3 activation in a rodent model of diabetic retinopathy. Diabetes 54(5):1559-1565. https://doi.org/10.2337/diabetes.54.5. 1559

34. van Hecke MV, Dekker JM, Nijpels G et al (2005) Inflammation and endothelial dysfunction are associated with retinopathy: the Hoorn Study. Diabetologia 48(7):1300-1306. https://doi.org/10. 1007/s00125-005-1799-y

35. Tang J, Kern TS (2011) Inflammation in diabetic retinopathy. Prog Retin Eye Res 30(5):343-358. https://doi.org/10.1016/j.preteyeres. 2011.05.002

36. Joussen AM, Murata T, Tsujikawa A, Kirchhof B, Bursell SE, Adamis AP (2001) Leukocyte-mediated endothelial cell injury and death in the diabetic retina. Am J Pathol 158(1):147-152. https://doi.org/10.1016/S0002-9440(10)63952-1

37. Jiang F, Chen Q, Huang L et al (2016) TNFSF15 inhibits blood retinal barrier breakdown induced by diabetes. Int J Mol Sci 17(5): 615. https://doi.org/10.3390/ijms17050615

38. Yun JH, Park SW, Kim KJ et al (2017) Endothelial STAT3 activation increases vascular leakage through downregulating tight junction proteins: implications for diabetic retinopathy. J Cell Physiol 232(5):1123-1134. https://doi.org/10.1002/jcp.25575

39. Behl T, Kotwani A (2015) Exploring the various aspects of the pathological role of vascular endothelial growth factor (VEGF) in diabetic retinopathy. Pharmacol Res 99:137-148. https://doi.org/ 10.1016/j.phrs.2015.05.013

40. Ding X, Gu R, Zhang M et al (2018) Microglia enhanced the angiogenesis, migration and proliferation of co-cultured RMECs. BMC Ophthalmol 18(1):249. https://doi.org/10.1186/s12886-018-0886-Z

41. Mitsuhashi J, Morikawa S, Shimizu K, Ezaki T, Yasuda Y, Hori S (2013) Intravitreal injection of erythropoietin protects against retinal vascular regression at the early stage of diabetic retinopathy in streptozotocin-induced diabetic rats. Exp Eye Res 106:64-73. https://doi.org/10.1016/j.exer.2012.11.001

42. Wang Q, Pfister F, Dorn-Beineke A et al (2010) Low-dose erythropoietin inhibits oxidative stress and early vascular changes in the experimental diabetic retina. Diabetologia 53(6):1227-1238. https://doi.org/10.1007/s00125-010-1727-7

43. Grimm C, Willmann G (2012) Hypoxia in the eye: a two-sided coin. High Alt Med Biol 13(3):169-175. https://doi.org/10.1089/ ham.2012.1031

44. Mana L, Wang S, Zhu H et al (2014) Qingkailing suppresses the activation of $\mathrm{BV}_{2}$ microglial cells by inhibiting hypoxia/ reoxygenation-induced inflammatory responses. Evid Based Complement Alternat Med 2014:696218. https://doi.org/10.1155/ 2014/696218

45. Kokona D, Ebneter A, Escher P, Zinkernagel MS (2018) Colonystimulating factor 1 receptor inhibition prevents disruption of the blood-retina barrier during chronic inflammation. J Neuroinflammation 15(1):340. https://doi.org/10.1186/s12974018-1373-4

46. Grieb P (2016) Intracerebroventricular streptozotocin injections as a model of Alzheimer's Disease: in search of a relevant mechanism. Mol Neurobiol 53(3):1741-1752. https://doi.org/10.1007/s12035015-9132-3

Publisher's note Springer Nature remains neutral with regard to jurisdictional claims in published maps and institutional affiliations. 